\title{
The frequency and rate of pilin antigenic variation in Neisseria gonorrhoeae
}

\author{
Alison K. Criss, Kimberly A. Kline ${ }^{\star}$, and H. Steven Seifert ${ }^{\dagger}$ \\ Department of Microbiology-Immunology, Northwestern University Feinberg School of Medicine
}

\section{Summary}

The pilin antigenic variation (Av) system of Neisseria gonorrhoeae (Gc) mediates unidirectional DNA recombination from silent gene copies into the pilin expression locus. A DNA sequencing assay was developed to accurately measure pilin Av in a population of Gc strain FA1090 arising from a defined pilin progenitor under nonselective culture conditions. This assay employs a piliated parental Gc variant with a recA allele whose promoter is replaced by lac-regulatory elements, allowing for controlled induction of pilin Av. From this assay, the frequency of pilin Av was measured as 0.13 recombination events per colony, with a corresponding rate of pilin Av of $4 \times 10^{-3}$ events per colony per generation. Most pilin variants retained the parental piliation phenotype, providing the first comprehensive analysis of piliated variants arising from a piliated progenitor. Sequence analysis of pilin variants revealed that a subset of possible recombination events predominated, which differed between piliated and nonpiliated progeny. Pilin Av exhibits the highest reported frequency of any pathogenic gene conversion system and can account for the extensive pilin variation detected during human infection.

\section{Keywords}

gonorrhea; recombination; pilus

\section{Introduction}

\begin{abstract}
Homologous recombination of DNA is a fundamental cellular process involved in replication, DNA repair, and the generation of genetic diversity. Gene conversion, the nonreciprocal incorporation of DNA sequence into a homologous locus, underlies immunoglobulin variation in some mammals (Knight, 1992) and is also used by pathogens such as Borrelia,

Campylobacter, Plasmodium, and Trypanosoma to antigenically vary virulence-associated surface structures (van der Woude and Baumler, 2004). Antigenic variation (Av) has been implicated in evasion of the host humoral immune response and adaptation to new intracellular niches or to different host species (Liang et al., 2004; Smith et al., 1995). During infection, frequencies of gene conversion range from $10^{-1}$ to $<10^{-4}$ recombinants per cell, with corresponding rates of $10^{-2}$ to $<10^{-5}$ recombinants per cell per generation (Horrocks et al., 2004; Keely et al., 2003; Roberts et al., 1992; Roske et al., 2001; Tu et al., 2003; Turner, 1997), but some of these frequencies may reflect selection in the host.
\end{abstract}

Pilin Av in the human-specific pathogen Neisseria gonorrhoeae (Gc) is one of the bestunderstood systems of gene conversion (Kline et al., 2003). Pilin is the major subunit of the

†Corresponding author. Address: 303 E. Chicago Ave. Searle 6-450, Mailcode S213, Chicago, IL 60611. Phone: (312) 503-9788. Fax: (312) 503-1339. E-mail: h-seifert@ northwestern.edu.

Present address: Department of Molecular Microbiology, Washington University School of Medicine, St. Louis, MO 63110. 
neisserial type IV pili, which are present on all clinical isolates of Gc and are essential for establishing urogenital infection (Cohen and Cannon, 1999). Gc carry a pilin expression locus, pilE, and multiple storage loci containing unexpressed pilin coding information, collectively termed pilS, on their chromosome. During pilin Av, part or all of a pilS copy is transferred unidirectionally into pile, giving rise to new pilin variants that differ in their ability to be expressed or assembled into pili. As a result, pilin Av confers a spectrum of pilusdependent colony morphologies on $\mathrm{Gc}$, from heavily piliated $(\mathrm{P}+)$ to nonpiliated $(\mathrm{P}-)$.

In the Gc strain FA1090, 19 unique pilS copies reside in 6 discrete chromosomal loci (Hamrick et al., 2001). All pilS copies lack the promoter, ribosomal binding site, and 5' $150 \mathrm{bp}$ found at pilE (Haas and Meyer, 1986; Haas et al., 1992; Segal et al., 1986). Based upon sequence divergence in the pilS repertoire, pilS copies are comprised of a 5' semivariable region, corresponding to the central $\sim 250$ bp of pilE, and a 3 ' hypervariable loop and tail $\left(\mathrm{HV}_{\mathrm{L}}\right.$ and $\mathrm{HV}_{\mathrm{T}}$ ) of $\sim 100 \mathrm{bp}$ in length. Within these variable regions are short (1-34 bp) regions of sequence conserved among all pilS copies and pilE thought to provide the homology that drives recombination. As with all homologous recombination pathways, RecA function is essential for pilin Av (Koomey et al., 1987). Pilin Av also requires a RecF-like pathway (RecQ, RecO, RecR, and RecJ), the RecA modulator RecX, the growth regulator RdgC, the Rep helicase and the Holliday junction processing enzymes RecG and RuvB (Kline and Seifert, 2005a; Mehr and Seifert, 1997, 1998; Mehr et al., 2000; Sechman et al., 2005; Skaar et al., 2002; Stohl and Seifert, 2001).

Estimates of the frequency of pilin Av are based upon P-colony appearance or sequence changes in the pile $\mathrm{HV}_{\mathrm{L}}$ and range from $10^{-2}$ to $10^{-4}$ variants per colony-forming unit (CFU) (Bergstrom et al., 1986; Koomey et al., 1987; Rohrer et al., 2005; Segal et al., 1985; Serkin and Seifert, 1998; Wainwright et al., 1994; Zhang et al., 1992). To directly measure pilin Av, we developed a DNA sequencing assay that nonselectively identifies pilin variants arising from a defined pilE progenitor. Pilin Av is controlled by the lac-inducible regulation of $r e c A$ expression, which allows for the frequency and rate of this process to be accurately measured. We have measured a frequency of pilin Av for FA1090 variant 1-81-S2 of 0.13 recombination events per CFU and a corresponding rate of $4.0 \times 10^{-3}$ events per CFU per generation, values that are 5-500 times higher than previously reported for this Gc variant. The large number of $\mathrm{P}+$ and $\mathrm{P}-$ pilin antigenic variants analyzed allowed for definition of the recombination events underlying pilin Av at a previously unavailable level of detail.

\section{Results}

\section{Measuring the frequency and rate of pilin Av with the pilE sequencing assay}

We developed a novel DNA sequencing-based assay to measure the total amount of pilin Av independent of colony morphology changes in a population of Gc. This assay depends upon three key factors. First, Gc of strain background FA1090 were used, since all pilS copies in this strain have been sequenced (Hamrick et al., 2001). Second, the specific FA1090 isolate used harbors the recA6 allele, in which RecA expression is controlled by lac-regulatory elements (Seifert, 1997). In the recA6 genetic background, RecA-dependent processes like pilin Av are initiated only when Gc are grown in the presence of IPTG (Rohrer et al., 2005; Serkin and Seifert, 1998; Seifert, 1997). The recA6 isolate encodes the 1-81-S2 pilin variant (Seifert et al., 1994), a highly piliated variant that has been used previously to measure pilin Av (Rohrer et al., 2005; Serkin and Seifert, 1998). The combination of the recA6 allele with the 1-81-S2 variant sequence ensures that the parental colonies uniformly express one pilin protein. Third, large-scale DNA sequencing allowed the pilE genes from a large number of randomly selected progeny to be rapidly examined. In combination, these factors yield the most complete analysis of pilin Av to date. 
To perform the assay, 3-7 single colonies of FA1090 1-81-S2 recA6 Gc are grown in the presence of IPTG to allow pilin Av to occur. These colonies are dispersed and grown on solid medium in the absence of IPTG. At least $48 \mathrm{CFU}$ arising from each parental colony are isolated and serially passaged to ensure the recovery of pure populations of Gc. The pilE loci of the progeny are sequenced and compared to both the parental pilE allele as well as the pilS copies present in the FA1090 genome. These numbers of starting and progeny colonies were determined statistically to yield accurate, reproducible measurements of pilin Av (see Experimental Procedures). The assay was performed with three independent cultures of Gc, generating a total of 497 progeny colonies that were examined for pilE sequence changes.

$5.2-17.3 \%$ of pilE clones in the three sequenced populations differed from 1-81-S2, averaging $11.8 \pm 3.5 \%$ variation. Strikingly, $0.96 \%$ of the pilE sequences in each replicate population had undergone two recombination events, comprising $13.3 \%$ of all nonparental pilE sequences. A recombination event is defined as the incorporation of new pilS sequence into pilE; when multiple recombination events have occurred, sequences corresponding to different pilS copies appear at various sites in pilE Detection of two recombination events in a pilE sequence was a reasonable expectation: if $10 \%$ of clones exhibit one event, $1 \%$ would on average exhibit two. However, multiple recombination events per pilE sequence have never before been taken into account when measuring pilin Av. Since the sequencing assay had the unique ability to identify these events, we redefined the frequency of pilin Av as the number of recombination events measured in a population divided by the total number of pilE genes sequenced, i.e. the number of CFU. In the $497 \mathrm{P}+\mathrm{CFU}$ examined, the frequency of pilin Av was $0.13 \pm 0.04$ recombination events per CFU. The DNA sequencing assay is therefore able to detect substantial amounts of pilin Av in a Gc population cultured under nonselective conditions.

Assuming each colony grown in the presence of IPTG arose from one CFU and contained 19 generations of bacteria $\left(\sim 3 \times 10^{5} \mathrm{CFU}\right.$ per colony), the resulting rate of pilin Av is $\sim 6.8 \times$ $10^{-3}$ recombination events per $\mathrm{CFU}$ per generation. In order to more accurately measure the rate of pilin Av, FA1090 1-81-S2 recA6 Gc were grown in liquid medium containing IPTG, and the frequency of pilin Av was analyzed at $3 \mathrm{hr}$ intervals up to $12 \mathrm{hr}$ after IPTG induction for three independent cultures. The average generation time of these cultures was $61 \mathrm{~min}$. No antigenic variants were detected in any of the replicate cultures at $3 \mathrm{hr}$ after IPTG induction (a frequency of $<9.52 \times 10^{-3}$ events per CFU). At $6 \mathrm{hr}$, the average frequency was $1.05 \times 10^{-2}$ events per CFU; at $9 \mathrm{hr}, 3.52 \times 10^{-2}$ events per CFU; at $12 \mathrm{hr}, 5.13 \times 10^{-2}$ events per CFU. Using the data from the 6 to $12 \mathrm{hr}$ time points, the rate of pilin Av was calculated to be $4.0 \pm$ $0.6 \times 10^{-3}$ recombination events per $\mathrm{CFU}$ per generation.

The results with FA1090 1-81-S2 recA6 were validated using Gc with a wild-type $r e c A$ allele, in which pilin Av occurs constitutively. Three experiments were conducted, yielding 180 pilE clones. $22.5 \pm 2.7 \%$ of progeny $\mathrm{CFU}$ encoded nonparental pilE sequences, with a corresponding frequency of pilin Av of $0.25 \pm 0.01$ recombination events per CFU. This frequency is within two fold of that measured in $r e c A 6$ Gc. The rate of pilin Av of Gc with a wild-type $r e c A$ allele was estimated by culturing Gc in liquid medium and performing the sequencing assay at $0,3,6,9$, and $12 \mathrm{hr}$ after inoculation. Since in this experiment the starting inoculum exhibited a frequency of pilin $\mathrm{Av}$ of 0.25 events per $\mathrm{CFU}$, the rate was calculated as the change in frequency at each time point divided by the number of generations and was found to be $1.8 \pm 0.4 \times 10^{-2}$ recombination events per CFU per generation. The frequency and rate calculations with this $\mathrm{Gc}$ strain are substantially higher than measured in $r e c A 6 \mathrm{Gc}$, but these values should only be considered estimates since the starting colonies of FA1090 1-81-S2 Gc may have contained bacteria with nonparental pilE sequence. Furthermore, it is likely that each wild-type $r e c A$ colony contains a different percentage of nonparental pilE variants, but since these variants arise stochastically, the basal level of variation in a colony cannot be accurately assessed. When the frequency of pilin Av is measured, the variant sequences present at the 
start are recorded as if they had arisen during the course of the experiment (although they were already present at the start), thus affecting the calculated rate. In contrast, at the time of recA induction the starting population of the recA6 Gc is uniform in its pilE sequence, which then produces a defined rate. These results show that the values calculated with $\mathrm{Gc}$ with a wild-type $r e c A$ allele are similar to those measured in the recA6 strain, and even after taking into account the variability inherent in wild-type colonies, may in fact be higher. We conclude that the pilE sequencing assay for Gc reproducibly measures pilin $\mathrm{Av}$, and it reveals that the frequency and rate of this process are significantly higher than previously reported (Rohrer et al., 2005; Serkin and Seifert, 1998).

\section{Nonrandom incorporation of pilS sequences in $\mathrm{P}+$ pilE variants}

Prior to this study, large-scale analysis of pilin antigenic variants was limited to Gc exhibiting pilus-dependent colony morphology changes. However, results from the pilE sequencing assays clearly demonstrate that a sizable percentage of $\mathrm{P}+\mathrm{Gc}$ arising from a $\mathrm{P}+$ progenitor undergo pilin Av. We therefore used the sequence information from these assays to investigate the recombination events arising in P+ variants of FA1090 1-81-S2. 93 nonparental pilE sequences were recovered from 1-81-S2 harboring either the recA6 or the wild-type recA allele. Five of the 93 pilE clones had undergone two recombination events at pilE, generating 98 total recombination events. 92 of the 98 events could be unambiguously assigned to one or two pilS copies. The remainder diverged from 1-81-S2 at 1-2 bp and corresponded to sequences found in multiple silent copies. It is likely that these single nucleotide changes are true antigenic variants and not point mutants, since a) the nucleotide changes are in variable regions and are present in several pilS copies that have flanking sequence identity to 1-81-S2, and b) no single nucleotide changes were detected elsewhere in pilE, including conserved sequences.

The $92 \mathrm{P}+$ recombination events with identifiable donor silent copies had sequence corresponding to 12 of the 19 pilS copies in Gc strain FA1090. Within this subset, certain silent copies predominated (Table 1). The most frequently recovered recombinant exhibited a change in the $\mathrm{HV}_{\mathrm{T}}$ region of pilE corresponding to the sequence found in both pilS2cl and pilS6cl

(Fig. 1). It cannot be determined whether one or both of these donors contributed to this recombination event, since both share sequence identity with 1-81-S2 pilE upstream of the $\mathrm{HV}_{\mathrm{T}}$. However, pilS2cl is identical to 1-81-S2 pilE for 268 bp upstream of the $\mathrm{HV}_{\mathrm{T}}$ (dotted line in $2 c 1 / 6 c 1$, Fig. 1), whereas pilS6cl only shares 32 bp of sequence with 1-81-S2 pilE in this region (solid line). Sequences from pilS3c1, pilS1c1, and pilS3c2 also appeared repeatedly. Notably, four recombination events, pilS2c1/6c1, pilS3c1-A and -B, and pilS3c2-A, accounted for $57 \%$ of the events detected (Fig. 1), and all arose in more than one of the six pilE sequencing assays. In total, eight pilE variants emerged in more than one of the sequencing assays performed (arrowheads, Fig. 1), indicating that certain recombination events are more frequently recovered than others. The most predominant pilS copies appeared at similar levels in both recA6 and wild-type recA genetic backgrounds, indicating there is no mechanistic difference between these two strains (data not shown). The relative frequency of incorporation of pilS copies into the pilE $\mathrm{HV}_{\mathrm{L}}$ differed from full-length pilE, but correlated with a previous examination of the repertoire of donor pilS sequences in the $\mathrm{HV}_{\mathrm{L}}$ arising from the 1-81-S2 variant (Rohrer et al., 2005).

All $98 \mathrm{P}+$ recombination events were examined for the length and position of variant sequence in pilE (Fig. 1).The length of contiguous variant sequence incorporated in pilE averaged $55 \mathrm{bp}$ and ranged from $1 \mathrm{bp}$ (A326G) to greater than $200 \mathrm{bp}$ (pilS3cl recombinant C, pilS1c5- B). Notably, $50 \%$ of the recombination events involved incorporation of $15 \mathrm{bp}$ or less of pils sequence ( $p i l S 2 c 1 / 6 c 1$, pilS3c2- A and -E, pilS3c3 A). In contrast, only one recombination event involved incorporation of an entire pilS copy (pilS7c1). The $98 \mathrm{P}+$ recombination events occurred throughout pilE, with $50 \%$ of variants exhibiting changes in the semivariable region. 
As previously observed (Hamrick et al., 2001;Howell-Adams and Seifert, 2000;Seifert, 1996), the location of the endpoints of variant information did not support the minicassette model of pilin Av (Haas and Meyer, 1986). Taken together, these results indicate that $\mathrm{P}+$ antigenic variants derived from 1-81-S2 pilE are generated from a subset of pilS copies that donate short stretches of sequence to pilE, and that a small number of pilin variants exhibiting identical recombination events at pilE arise repeatedly from this progenitor pilin sequence.

\section{Generation of P- variants by pilin Av}

Gc colonies of P- morphology arise from pilin Av, L-pilin production (the incorporation of multiple pilS copies in tandem into pilE, yielding an aberrantly long, nonfunctional protein), deletion of the pilE locus, and phase variation of the structural PilC proteins (Hagblom et al., 1985; Jonsson et al.., 1991; Segal et al., 1985; Swanson et al., 1985; Swanson et al., 1986). To explore the relative contribution of these events to P- colony formation, FA1090 1-81-S2 recA6 was grown on solid medium containing IPTG to induce pilin Av, sectors of colonies exhibiting a less piliated morphology were isolated, and the pilE genes were sequenced from progeny retaining the $\mathrm{P}$ - morphology.Three replicate experiments were performed with a total of 67 P- CFU analyzed.

The appearance of $\mathrm{P}$ - colonies in a population has been used as a surrogate measure of pilin Av, and we observed that $58.6 \%$ of the P- clones had undergone pilin Av. The remaining $41.4 \%$ of P- colonies exhibited one of three phenotypes: 1) production of L-pilin, determined by the generation of an abnormally long PCR product using pilE-specific primers $(6.5 \%$ of the Pisolates); 2) deletion of the pilE locus, assessed by the inability to produce a PCR product with these same pilE-specific primers (20.2\%); and 3) PilC phase variation, or the retention of parental pilE sequence while exhibiting P-morphology (14.7\%). The absence of PilC expression was confirmed by Western blot (data not shown). Two of the $67 \mathrm{P}-\mathrm{CFU}$ exhibited sequence changes that were detected in the $\mathrm{P}+$ population and were also PilC phase variants $(2 c 1 / 6 c l$ and $1 c 5$, Fig. 2). These results demonstrate that pilin Av is a common mechanism for generating P- variants, but other mechanisms are of equal prevalence and should be taken into consideration when using phase variation as a surrogate measure of pilin Av.

The 47 recA6 and 5 wild-type $r e c A$ P- pilin antigenic variants yielded a total of $52 \mathrm{P}$ - variants and 64 recombination events. 61 of the 64 recombination events could be assigned to one or two pilS copies (Fig. 2). Compared to the P+ variants, a smaller and different subset of pilS copies were represented in the P- variants (Table 1). As with the P+ variants, the most predominant pilS copies appeared in each of the replicate P-population at a similarly high frequency (data not shown). These variants fell into two categories. The first class of variants, accounting for $7 \%$ of the P- sequences, was predicted to produce full-length pilin protein but produced a P- colony phenotype. These variants are classified as "assembly mutants," where pilin monomers are not effectively assembled into the growing pilus (Swanson et al., 1986). No pilin protein was detected in these variants by Western blot, but PilC was still expressed (data not shown). This class of variants incorporated pilS2cl or pilS6cl in the semivariable region of pilE (2cl-A and -B and $6 c 1-\mathrm{A}$ and -B, Fig. 2).

The majority of P- pilin variants also failed to react with pilin-specific antiserum, but these variants were predicted to encode truncated proteins. These $\mathrm{P}$ - variants contained sequence encoded in one of 4 pilS copies: pilS2c4, pilS3c2, pilS6c2, and pilS1c3 (Fig. 3). Incorporation of pilS2c4, pilS3c2, and pilS6c2 at the 5' end of the pilE semivariable region generates a stop codon after residue 94 or 95 , and incorporation of pilS1c3 introduces a stop codon at position 78.3 of the 4 pilS copies contain poly(C)tracts that could vary in length as a result of replication errors to restore a P+ phenotype (Koomey et al., 1987). As with P+ variants, certain pilS sequences in these $\mathrm{P}$ - variants predominated, with pilS2c4 and pilS3c2 detected more frequently than the other two (Table 1). 
Compared to $\mathrm{P}+$ variants, $\mathrm{P}-$ variants on average contained longer tracts of pilS sequence which was incorporated closer to the 5' end of the pilE semivariable region (Fig. 2). 86\% of Precombination events occurred in the semivariable region of pilE, and the average minimum length of pils sequence incorporation in P- variants was $133 \mathrm{bp}$. Although pilS incorporation as short as 1 bp could be detected (C395A, Fig. 2), only 15.7\% of variants had incorporated. $15 \mathrm{bp}$ or less of pilS sequence. In summary, pilin Av accounts for the majority of P-colonies generated after in vitro passage of $\mathrm{Gc}$, and these variants arise from recombination with a subset of pilS copies that encode truncated or poorly assembled pilin proteins.

\section{Minimal sequence identity is required for pilin Av}

RecA-dependent homologous recombination requires sequence identity between donor and recipient DNA of at least 73 bp (Nash, 1996). Each donor pilS copy identified in the variant pilE population was aligned with 1-81-S2 pilE to identify regions of shared sequence (depicted for a subset of pilS copies in Figure 1 and Figure 2). For each recombination event where the donor pilS copy could be unambiguously identified, the length of identical sequence between the incoming copy and 1-81-S2 pilE flanking the recombinant tract was measured. The median amount of shared sequence was $44 \mathrm{bp}$ at the 5' flanking region and $39 \mathrm{bp}$ at the 3 ' flaning region, and 8 independent recombination events were detected where shared sequence identity was 2-4 bp. No significant difference was noted in the amount of sequence overlap between $\mathrm{P}+$ and $\mathrm{P}-$ variants. Minimal regions of shared sequence identity were not necessarily paired with especially extensive sequence identity at the other end of the recombination tract, and the length of sequence overlap was not related to the length of pilS sequence appearing in pilE. This analysis confirms that minimal sequence identity is needed for pilE recombination (Howell-Adams and Seifert, 2000) and further demonstrates that recombination is independent of the length or identity of the pilS sequence incorporated, lending further support for the current understanding of pilin Av as a specialized form of homologous recombination.

\section{Discussion}

Gonococcal pilin Av is a prototype for prokaryotic gene conversion systems, yet a direct measure of the frequency and rate of this process has never been reported. We developed a pilE sequencing assay to address this issue, which revealed that 1 out of 4-8 CFU in a population has undergone recombination at $p i l E$. These frequencies are three- to ten-fold higher than measured with a RT-PCR assay examining variants in the $\mathrm{HV}_{\mathrm{L}}$, the most complete assay for pilin Av until this point (Rohrer et al., 2005; Serkin and Seifert, 1998).Strikingly, the maximum frequency of pilin Av may be even higher than reported here, since FA1090 Gc with a different starting pilE sequence was found to produce a greater percentage of $\mathrm{HV}_{\mathrm{L}}$ variants than 1-81-S2 (Rohrer et al.., 2005). To our knowledge, pilin Av exhibits the highest frequency of gene conversion of any pathogenic antigenic variation system examined to this point (van der Woude and Baumler, 2004). One drawback to this assay is its cost, considering that a minimum of 480 PCR products need to be sequenced per time point for a given strain. However, this assay demonstrated that most antigenic variants retain the parental $\mathrm{P}+$ phenotype and provided the first comprehensive analysis of $\mathrm{P}+$ variants arising from a $\mathrm{P}+$ progenitor. It also identified a novel means by which $\mathrm{P}-$ variants arise from a $\mathrm{P}+$ progenitor. Finally, by generating 159 nonparental pilE sequences encompassing 48 unique pilin variants (Supplemental Fig. 1), the assay produced the most complete picture available about the recombination events of pilin Av.

It is apparent from the $\mathrm{P}+$ and $\mathrm{P}-$ variant pilE sequences arising from FA1090 1-81-S2 that certain pils copies predominate, even in the absence of any apparent selection pressure. We have calculated that 963 independent antigenic variants could arise following one recombination event with 1-81-S2 pile, if at least $2 \mathrm{bp}$ of sequence identity exists at the end 
of a tract of new pilE sequence. This is inconsistent with the observation that only 3 recombination events account for 50\% of the $\mathrm{P}+$ variant population $(2 c 1 / 6 c 1$ and $3 c 1-\mathrm{A}$ and -B, Fig. 1). These are not isolated events, since these and other high-frequency recombinants occurred in multiple experiments (arrowheads, Fig. 1). Several explanations for the nonrandom distribution of pilS donors were considered, including the chromosomal location of a pilS copy or its position in the pilS locus, its relative percentage of sequence identity to pilE, the presence of pilin-associated repeat elements (Hamrick et al., 2001), and the length of the sequence tracts flanking the pilE/pilS recombinants. The antigenic variation data presented in this work does not individually support any of these possibilities. We postulate that either a combination of these factors dictates the prevalence of pilE recombination events, or some as-yet unknown sequence element or bacterial factor influences the frequency of pilS recombination at the pilE locus.

In FA1090 1-81-S2, the predominant mechanism for generating P- variants was recombination with silent copies that shift the pilE coding frame and prematurely incorporate a stop codon. These truncations were predicted from the sequence analysis of pilS copies by Hamrick $e t$ al. (Hamrick et al., 2001) but were not previously recovered in P- Gc. It is important to note that these variants differ from S-pilin variants, which encode full-length pilin proteins that undergo posttranslational removal of their N-termini (Koomey et al.., 1991). Although we have no direct evidence that $\mathrm{P}$ - variants occur at a measurable frequency during infection, the retention of pilS copies encoding truncated pilins in the genome suggests that nonpiliated variants may have a role in infection. The first possibility for such a role is that nonpiliated Gc have a growth advantage in vivo since, in vitro, nonpiliated Gc have an enhanced metabolic rate compared to an isogenic piliated strain and can outgrow their piliated counterparts when cultured together in liquid medium (H.S. Seifert, unpublished results). The second possibility is that loss of piliation is advantageous during infection. For instance, the reduced adherence capacity of nonpiliated bacteria may facilitate Gc movement within a persor or transfer between individuals. Furthermore, P- Gc would not be recognized by host pilin-specific antibodies, and in this light a lack of piliation can be considered an extreme form of Av. The third possibility is that nonpiliated Gc may alter host cell signaling. In epithelial cells, neisserial pili and their retractile forces induce $\mathrm{Ca}^{2+}$ spikes and the activation of protein kinases (Howie et al., 2005; Kallstrom et al., 1998); modulation of these signals by P- Gc could affect, for example, the inflammatory response. If any or all of these possibilities are found to be important for Gc pathogenesis, pilin Av would provide the high-frequency mechanism behind $\mathrm{P}+\leftrightarrow \mathrm{P}$ -variation.

The extent of pilin Av measured by the DNA sequencing assay can be compared to that reported during human infection. The majority of Gc recovered from experimentally infected male volunteers encode nonparental pilE sequences after $24 \mathrm{hr}$ of infection, and some pilE genes contain more than one recombination tract (Hamrick et al., 2001; Seifert et al., 1994; Swanson et al.., 1987). These high levels of pilin variation were thought to be due either to stimulation of the frequency of pilin Av or selection against the parental pilin protein. In contrast, results from the sequencing assay would argue that the frequency of pilin Av in vitro is sufficient to account for all pilin variation that occurs during infection. It is not possible to directly compare the rate of pilin Av measured in vitro to that occurring in vivo because the number of generations of growth in the host cannot be determined: colonization bottlenecks occur early after inoculation that limit the number of bacteria that actually establish infection (Cohen and Cannon, 1999). Assuming all the progeny arising during acute infection were descended from one parental CFU and an in vitro rate of pilin Av, after one day approximately $50 \%$ of isolates would have undergone a recombination event, which is similar to what has been observed in experimentally infected individuals. Our results also correlate with the observation made in human volunteer studies that a small population of antigenic variants, involving a subset of pilS copies, predominate, without needing to invoke selection in vivo (Hamrick et al., 2001; 
Seifert et al., 1994). However, it is likely that in natural, long-term infections, immune selection is important for driving the appearance of a subpopulation of pilin variants. Since each newly arising pilin variant would give rise to its own unique repertoire of pilin sequences (Rohrer $e t$ al.., 2005), this ever-expanding pool of variants could facilitate immune evasion as Gc are transmitted within a sexual network and back to previously exposed individuals. We conclude that all observations made about gonococcal pilin Av in vitro are sufficient to explain the repertoire of pilin variants arising during infection, underscoring the importance of this process to the persistence and spread of Gc in the human population.

\section{Experimental Procedures}

\section{Bacterial strains and growth conditions}

1-81-S2 is a P+ variant of Gc strain FA1090 arising after experimental infection of a male volunteer (Seifert et al., 1994). FA1090 1-81-S2 recA6 contains an isopropyl $\beta$-Dthiogalactopyranoside (IPTG)-regulatable Gc recA gene, which allows for control of recA expression. These strains are phenotypically RecA-when IPTG is absent from the growth medium and phenotypically RecA+ in the presence of IPTG (Seifert, 1997). Gc were grown on GC Medium Base (Difco) plus Kellogg supplements (Kellogg et al., 1963) (GCB) at $37^{\circ} \mathrm{C}$ in $5 \% \mathrm{CO}_{2}$ for $20 \mathrm{~h}$, with the addition of $1 \mathrm{mM}$ IPTG (Diagnostic Chemicals Limited) when necessary. Colonies were observed in the stereomicroscope to ensure retention of the desired piliation phenotype. Liquid growth of Gc was performed in Gc liquid medium containing Kellogg's supplements and $0.042 \% \mathrm{Na}_{2} \mathrm{HCO}_{3}(\mathrm{GCBL})$ as described (Serkin and Seifert, 1998).

\section{Pilin variation assays}

(i).Growth on solid medium-The pilin variation assay was performed as described (Kline and Seifert, 2005b). 3-7 colonies of FA1090 1-81-S2 recA6 Gc grown on GCB-IPTG were each passaged on GCB. Approximately $48 \mathrm{P}+$ colonies that arose from each starter colony were passaged two more times to ensure clonal populations, then stored at $-80^{\circ} \mathrm{C}$. For Gc with the wild-type $r e c A$ allele, $1 \mathrm{P}+$ colony of Gc with 1-81-S2 pilE sequence was passaged on GCB. 48-96 colony-forming units (CFU) of both $\mathrm{P}+$ and $\mathrm{P}-$ colony morphology were collected and stored at $-80{ }^{\circ} \mathrm{C}$ without further passage. $\mathrm{P}$ - colonies comprised $\sim 5 \%$ of this population.

To examine P- variants in FA1090 1-81-S2 recA6, P- outgrowths arising from $\mathrm{P}+$ colonies on GCB-IPTG were isolated and streak purified for several days on GCB to ensure a homogeneous $\mathrm{P}$ - population was obtained. In each experiment, up to 32 independent $\mathrm{P}$ - clones were examined.

(ii) Growth in liquid medium-Liquid-grown FA1090 1-81-S2 recA6 were diluted into GCBL containing $1 \mathrm{mM} \mathrm{IPTG}$ at $37^{\circ} \mathrm{C}$. At $3,6,9$, and $12 \mathrm{~h}$ after IPTG induction, an aliquot of the culture was serially diluted and plated. The number of CFU present at each time point was determined, and $96 \mathrm{CFU}$ were collected from each time point. The experiment was performed in triplicate.

(iii) Statistical calculations-To determine the minimum sample size necessary to yield an accurate measurement of pilin Av, we performed a small pilot study with three 1-81-S2 recA6 starter colonies and 16-24 sequenced progeny from each, which had a standard deviation of $2.8 \%$ (data not shown). The formula

$$
\mathrm{N}=((\mathrm{z} * \text { s.d. }) / \mathrm{E})^{2}
$$


was then applied, where $\mathrm{N}=$ sample size, $\mathrm{z}=$ value corresponding to the confidence interval, s.d $=$ standard deviation of the sample, and $E=$ acceptable margin of error for the assay. Using $\mathrm{Z}=95 \%$ confidence interval and $\mathrm{E} \leq 1 \%$ margin of error, $\mathrm{N}$ was calculated at 48 progeny per starter colony.

pilE sequence analysis-The pilE locus was amplified by polymerase chain reaction (PCR) from Gc lysates as described previously (Kline and Seifert, 2005b). Sequencing reactions were carried out with the CEQ Dye Terminator Cycle Sequencing Quick Start Kit using primers specific for pilE and were run on a CEQ 2000XL automated sequencer (Beckman Coulter) according to the manufacturer's instructions. Alternatively, purified PCR products were sequenced commercially (SeqWright, Houston, TX).

Calculations for pilin antigenic variation-The pilE sequence from each clone was aligned with the parental 1-81-S2 pilE, as well as with all pilS copies present in the FA1090 genome (Hamrick et al.., 2001), using the AlignX program of the Vector NTI software (Informax, Inc.). In all cases, sequence changes observed in the isolates corresponded to one or more pilS copies. Nonparental pilE sequences were translated using the EditSeq program in the Lasergene software package (DNAStar, Inc.).

Each independent incorporation of pilS sequence into a pilE clone was designated a recombination event. For FA1090 recA6 Gc, the frequency of pilin Av was calculated by the following formula: Frequency $=$ Total number of recombination events at pilE $\div$ Total number of isolates sequenced. For FA1090 1-81-S2 Gc, the frequency of pilin Av was determined separately for $\mathrm{P}+$ and $\mathrm{P}-\mathrm{CFU}$, and the overall frequency was calculated by multiplying the frequency for each population by the fraction of colonies with the given colony morphology. Data are presented as the average of the frequencies measured from replicate samples \pm SEM. The rate of pilin Av is presented as the average change in frequency divided by the average number of bacterial generations over the course of the experiment \pm SEM.

Analysis of PilC and pilin production in P- variants-Lysates of P- variants were separated by SDS-polyacrylamide gel electrophoresis (10\% polyacrylamide for PilC, $15 \%$ for pilin) and transferred to polyvinylidene difluoride (Millipore). PilC was detected using a polyclonal anti-PilC antiserum (gift of J. Pfeifer and S. Normark) (Nassif et al., 1994) and pilin detected using T36 polyclonal antiserum (gift of M. So) (Forest et al., 1996), with both followed by goat anti-rabbit IgG coupled to horseradish peroxidase (Chemicon). Blots were developed using the ECLPlus chemiluminescence reagent (Amersham).

\section{Supplementary Material}

Refer to Web version on PubMed Central for supplementary material.

\section{Acknowledgments}

This work was supported by Public Health Service grants R37 AI033493 and R01 AI044239 to H.S.S. and Ruth L. Kirchstein National Research Service Award F32 AI056681 to A.K.C.

\section{References}

Bergstrom S, Robbins K, Koomey JM, Swanson J. Piliation control mechanisms in Neisseria gonorrhoeae. Proc Natl Acad Sci USA 1986;83:3890-3894. [PubMed: 2872674]

Cohen MS, Cannon JG. Human experimentation with Neisseria gonorrhoeae: progress and goals. J Infect Dis 1999;179:S375-S379. [PubMed: 10081510] 
Forest KT, Bernstein SL, Getzoff ED, So M, Tribbick G, Geysen HMX, Deal CD, Tainer JA. Assembly and antigenicity of the Neisseria gonorrhoeae pilus mapped with antibodies. Infect Immun 1996;64:644-652. [PubMed: 8550220]

Haas R, Meyer TF. The repertoire of silent pilus genes in Neisseria gonorrhoeae: evidence for gene conversion. Cell 1986;44:107-115. [PubMed: 2866848]

Haas R, Veit S, Meyer TF. Silent pilin genes of Neisseria gonorrhoeae MS11 and the occurrence of related hypervariant sequences among other gonococcal isolates. Mol Microbiol 1992;6:197-208. [PubMed: 1347637]

Hagblom P, Segal E, Billyard E, and So M. Intragenic recombination leads to pilus antigenic variation in Neisseria gonorrhoeae. Nature 1985;315:156-158. [PubMed: 2859529]

Hamrick TS, Dempsey JA, Cohen MS, Cannon JG. Antigenic variation of gonococcal pilin expression in vivo: analysis of the strain FA1090 pilin repertoire and identification of the pilS gene copies recombining with pilE during experimental human infection. Microbiology 2001;147:839-849. [PubMed: 11283280]

Horrocks P, Pinches R, Christodoulou Z, Kyes SA, Newbold CI. Variable var transition rates underlie antigenic variation in malaria. Proc Natl Acad Sci USA 2004;101:11129-11134. [PubMed: 15256597]

Howell-Adams B, Seifert HS. Molecular models accounting for the gene conversion reactions mediating gonococcal pilin antigenic variation. Mol Microbiol 2000;37:1146-1159. [PubMed: 10972832]

Howie HL, Glogauer M, So M. The N. gonorrhoeae type IV pilus stimulates mechanosensitive pathways and cytoprotection through a pilT-dependent mechanism. PLoS Biol 2005;3:e100. [PubMed: 15769184]

Jonsson AB, Nyberg G, Normark S. Phase variation of gonococcal pili by frameshift mutation in pilC, a novel gene for pilus assembly. EMBO J 1991;10:477-488. [PubMed: 1671354]

Kallstrom H, Islam MS, Berggren PO, Jonsson AB. Cell signaling by the type IV pili of pathogenic Neisseria. J Biol Chem 1998;273:21777-21782. [PubMed: 9705315]

Keely SP, Cushion MT, Stringer JR. Diversity at the locus associated with transcription of a variable surface antigen of Pneumocystis carinii as an index of population structure and dynamics in infected rats. Infect Immun 2003;71:47-60. [PubMed: 12496148]

Kellogg DS Jr, Peacock WL, Deacon WE, Brown L, Pirkle CI. Neisseria gonorrhoeae. I. Virulence genetically linked to clonial variation. J Bacteriol 1963;85:1274-1279. [PubMed: 14047217]

Kline KA, Sechman EV, Skaar EP, Seifert HS. Recombination, repair and replication in the pathogenic Neisseriae: the 3 R's of molecular genetics of two human-specific bacterial pathogens. Mol Microbiol 2003;50:3-13. [PubMed: 14507359]

Kline KA, Seifert HS. Role of the Rep helicase gene in homologous recombination in Neisseria gonorrhoeae. J Bacteriolc 2005a;187:2903-2907.

Kline KA, Seifert HS. Mutation of the priA gene of Neisseria gonorrhoeae affects DNA transformation and DNA repair. J Bacteriol 2005b;187:5347-5355. [PubMed: 16030229]

Knight KL. Restricted VH gene usage and generation of antibody diversity in rabbit. Annu Rev Immunol 1992;10:593-616. [PubMed: 1590997]

Koomey M, Gotschlich EC, Robbins K, Bergstrom S, Swanson J. Effects of recA mutations on pilus antigenic variation and phase transitions in Neisseria gonorrhoeae. Genetics 1987;117:391-398. [PubMed: 2891588]

Koomey M, Bergstrom S, Blake M, Swanson J. Pilin expression and processing in pilus mutants of Neisseria gonorrhoeae: critical role of Gly-1 in assembly. Mol Microbiol 1991;5:279-287. [PubMed: 1674976]

Liang FT, Yan J, Mbow ML, Sviat SL, Gilmore RD, Mamula M, Fikrig E. Borrelia burgdorferi changes its surface antigenic expression in response to host immune responses. Infect Immun 2004;72:57595767. [PubMed: 15385475]

Mehr IJ, Seifert HS. Random shuttle mutagenesis: gonococcal mutants deficient in pilin antigenic variation. Mol Microbiol 1997;23:1121-1131. [PubMed: 9106204]

Mehr IJ, Seifert HS. Differential roles of homologous recombination pathways in Neisseria gonorrhoeae pilin antigenic variation, DNA transformation, and DNA repair. Mol Microbiol 1998;30:697-710. [PubMed: 10094619] 
Mehr IJ, Long CD, Serkin CD, Seifert HS. A homologue of the recombination-dependent growth gene, $r d g C$, is involved in gonococcal pilin antigenic variation. Genetics 2000;154:523-532. [PubMed: 10655208]

Nash, HA. Site-specific recombination: integration, excision, resolution, and inversion of defined DNA segments. In: Neidhardt, FC., editor. Escherichia coli and Salmonella typhimurium: Cellular and Molecular Biology. Vol. Vol. 2. Washington D.C: American Society for Microbiology; 1996. p. 2363-2376.

Nassif X, Beretti JL, Lowy J, Stenberg P, O'Gaora P, Pfeifer J, Normark S, So M. Roles of pilin and PilC in adhesion of Neisseria meningitidis to human epithelial and endothelial cells. Proc Natl Acad Sci USA 1994;91:3769-3773. [PubMed: 7909606]

Roberts DJ, Craig AG, Berendt AR, Pinches R, Nash G, Marsh K, Newbold CI. Rapid switching to multiple antigenic and adhesive phenotypes in malaria. Nature 1992;357:689-692. [PubMed: 1614515]

Rohrer MS, Lazio M, Seifert HS. A real-time, semi-quantitative RT-PCR assay demonstrates that the pilE sequence dictates the frequency and characteristics of pilin antigenic variation in Neisseria gonorrhoeae. Nucleic Acids Res 2005;33:3363-3371. [PubMed: 15947134]

Roske K, Blanchard A, Chambaud I, Citti C, Helbig JH, Prevost MC, Rosengarten R, Jacobs E. Phase variation among major surface antigens of Mycoplasma penetrans. Infect Immun 2001;69:76427651. [PubMed: 11705944]

Sechman EV, Rohrer MS, Seifert HS. A genetic screen identifies genes and sites involved in pilin antigenic variation in Neisseria gonorrhoeae. Mol Microbiol 2005;57:468-483. [PubMed: 15978078]

Segal E, Billyard E, So M, Storzbach S, Meyer TF. Role of chromosomal rearrangement in N. gonorrhoeae pilus phase variation. Cell 1985;40:293-300. [PubMed: 2857113]

Segal E, Hagblom P, Seifert HS, So M. Antigenic variation of gonococcal pilus involves assembly of separated silent gene segments. Proc Natl Acad Sci USA 1986;83:2177-2181. [PubMed: 2870495]

Seifert HS, Wright CJ, Jerse AE, Cohen MS, Cannon JG. Multiple gonococcal pilin antigenic variants are produced during experimental human infections. J Clin Invest 1994;93:2744-2749. [PubMed: 7911129]

Seifert HS. Questions about gonococcal pilus phase- and antigenic variation. Mol Microbiol 1996;21:433-440. [PubMed: 8866467]

Seifert HS. Insertionally inactivated and inducible recA alleles for use in Neisseria. Gene 1997;188:215220. [PubMed: 9133594]

Serkin CD, Seifert HS. Frequency of pilin antigenic variation in Neisseria gonorrhoeae. J Bacteriol 1998;180:1955-1958. [PubMed: 9537399]

Skaar EP, Lazio MP, Seifert HS. Roles of the recJ and recN genes in homologous recombination and DNA repair pathways of Neisseria gonorrhoeae. J Bacteriol 2002;184:919-927. [PubMed: 11807051]

Smith JD, Chitnis CE, Craig AG, Roberts DJ, Hudson-Taylor DE, Peterson DS, Pinches R, Newbold CI, Miller LH. Switches in expression of Plasmodium falciparum var genes correlate with changes in antigenic and cytoadherent phenotypes of infected erythrocytes. Cell 1995;82:101-110. [PubMed: 7606775]

Stohl EA, Seifert HS. The recX gene potentiates homologous recombination in Neisseria gonorrhoeae. Mol Microbiol 2001;40:1301-1310. [PubMed: 11442829]

Swanson J, Bergstr:om S, Barrera O, Robbins K, Corwin D. Pilus- gonococcal variants. Evidence for multiple forms of piliation control. J Exp Med 1985;162:729-744. [PubMed: 2410533]

Swanson J, Bergstrom S, Robbins K, Barrera O, Corwin D, Koomey JM. Gene conversion involving the pilin structural gene correlates with pilus+ in equilibrium with pilus- changes in Neisseria gonorrhoeae. Cell 1986;47:267-276. [PubMed: 2876777]

Swanson J, Robbins K, Barrera O, Corwin D, Boslego J, Ciak J, Blake M, Koomey JM. Gonococcal pilin variants in experimental gonorrhea. J Exp Med 1987;165:1344-1357. [PubMed: 3106555]

Tu ZC, Wassenaar TM, Thompson SA, Blaser MJ. Structure and genotypic plasticity of the Campylobacter fetus sap locus. Mol Microbiol 2003;48:685-698. [PubMed: 12694614] 
Turner CM. The rate of antigenic variation in fly-transmitted and syringe-passaged infections of Trypanosoma brucei. FEMS Microbiol Lett 1997;153:227-231. [PubMed: 9252591]

van der Woude MW, Baumler AJ. Phase and antigenic variation in bacteria. Clin Microbiol Rev 2004;17:581-611. [PubMed: 15258095]

Wainwright LA, Pritchard KH, Seifert HS. A conserved DNA sequence is required for efficient gonococcal pilin antigenic variation. Mol Microbiol 1994;13:75-87. [PubMed: 7984095]

Zhang QY, DeRyckere D, Lauer P, Koomey M. Gene conversion in Neisseria gonorrhoeae: evidence for its role in pilus antigenic variation. Proc Natl Acad Sci USA 1992;89:5366-5370. [PubMed: 1351681] 


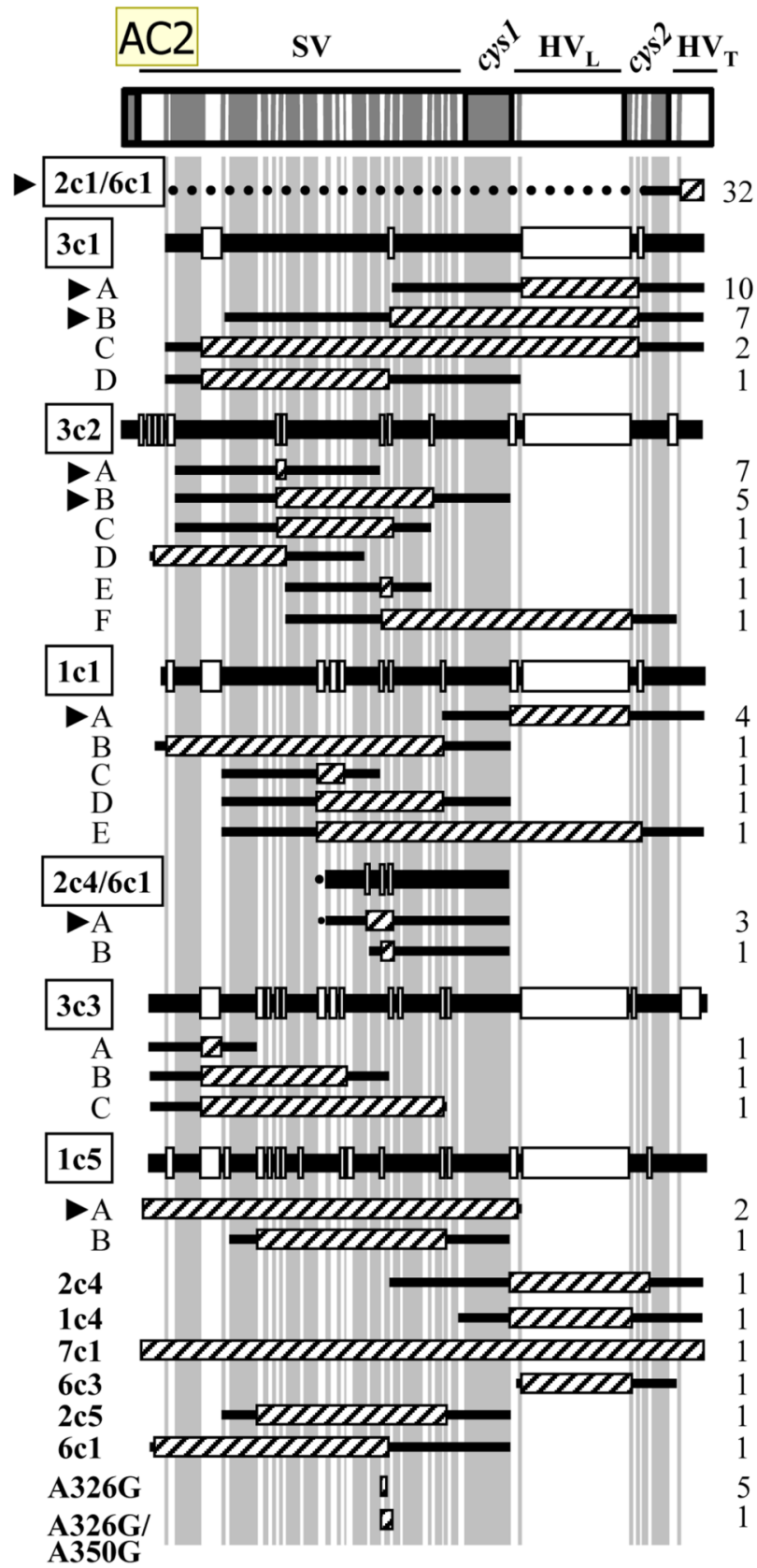

Figure 1. Recombination events in P+ variants of Gc strain FA1090 pils copies are abbreviated as in Table 1 . The positions in pilE of the semivariable (SV), hypervariable loop $\left(\mathrm{HV}_{\mathrm{L}}\right)$ and tail $\left(\mathrm{HV}_{\mathrm{T}}\right)$, and conserved cys 1 and cys 2 sequences are indicated. Sequences conserved among all pilS copies are gray and divergent regions are white; both are to scale. Recombination events involving the same donor pilS copy are grouped together and are presented in decreasing order of frequency of appearance in pilE. Hatched boxes indicate recombinant pilS sequence in 1-81-S2, and flanking sequence shared by 1-81-S2 and the donor pilS copy is indicated by flanking black lines. Six pilS copies (boxed) participated in more than one recombination event at pilE (labeled $\mathrm{A}, \mathrm{B}$, etc.). The alignment of each pilS copy with the 1-81-S2 sequence is shown above the corresponding recombination events; regions of each 
pilS copy that share identity with 1-81-S2 are indicated by black bars and divergent sequences by white boxes. In recombination events where two pilS copies could have served as donor $(2 c 4 / 6 c 1$ and $2 c 1 / 6 c 1)$, flanking sequence shared with both copies is indicated with a black line; sequence shared with only one copy is indicated by a dotted line. In pilS2cl/6cl, the dotted line represents additional homology between pilS2c1 and 1-81-S2 not present in pilS6c1; in pilS2c4, the dotted line represents additional homology between pilS6c1 and 1-81-S2 not present in pilS2c4. The number of times each recombination event was recovered is indicated at the right. The eight pilin variants recovered in independent cultures of Gc are indicated by arrowheads. A326G and A326G/A350G are sequences found in multiple pilS copies. 


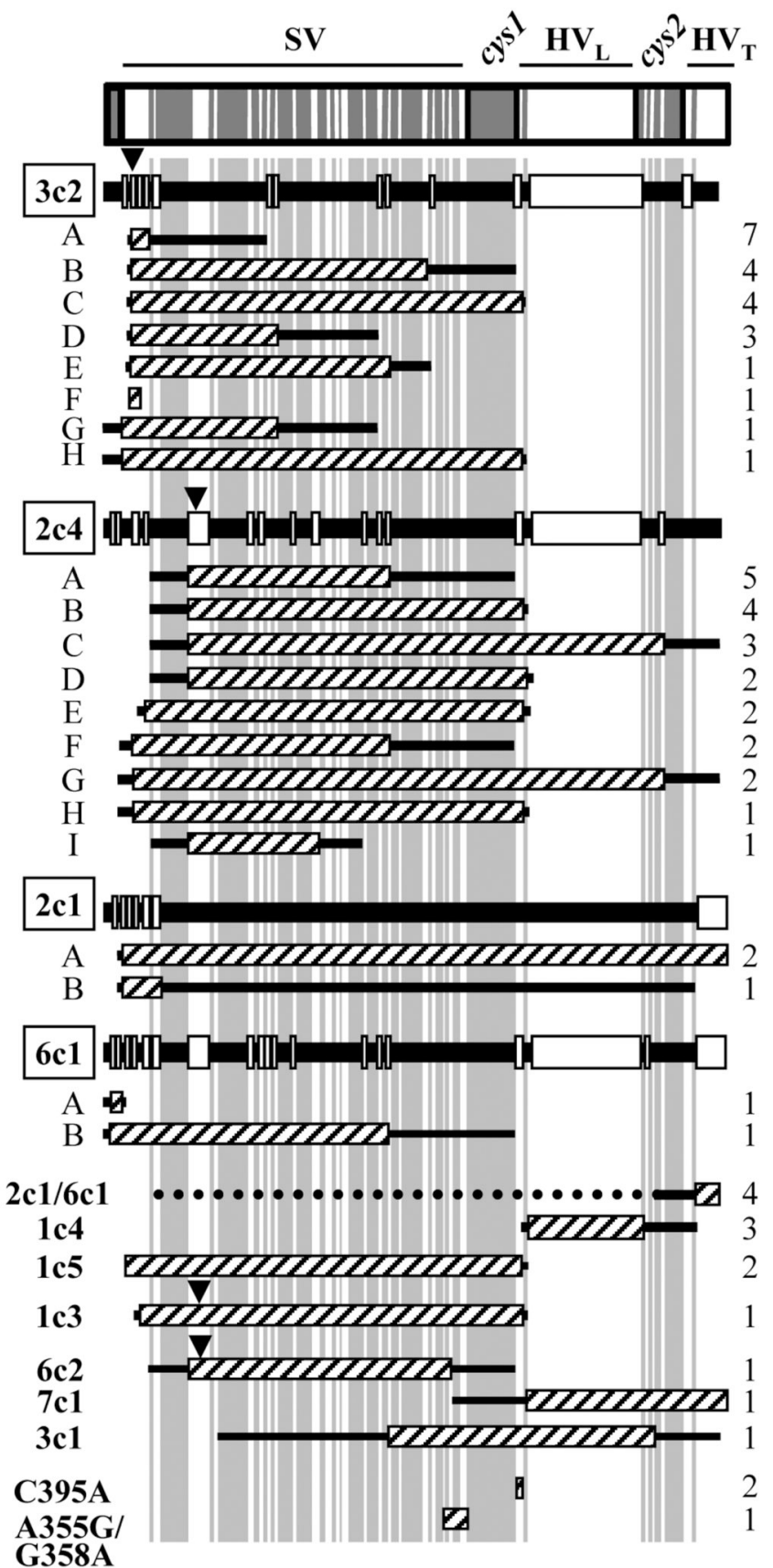

Figure 2. Recombination events in P- variants of Gc strain FA1090

Labeling of divergent and conserved regions, and alignments of pils copies with pilE, is as in Fig. 1. C395A and A355G/G358A are sequences found in multiple pilS copies. Black arrowheads indicate the position of frameshifts that are predicted to cause premature truncation of pilin. 


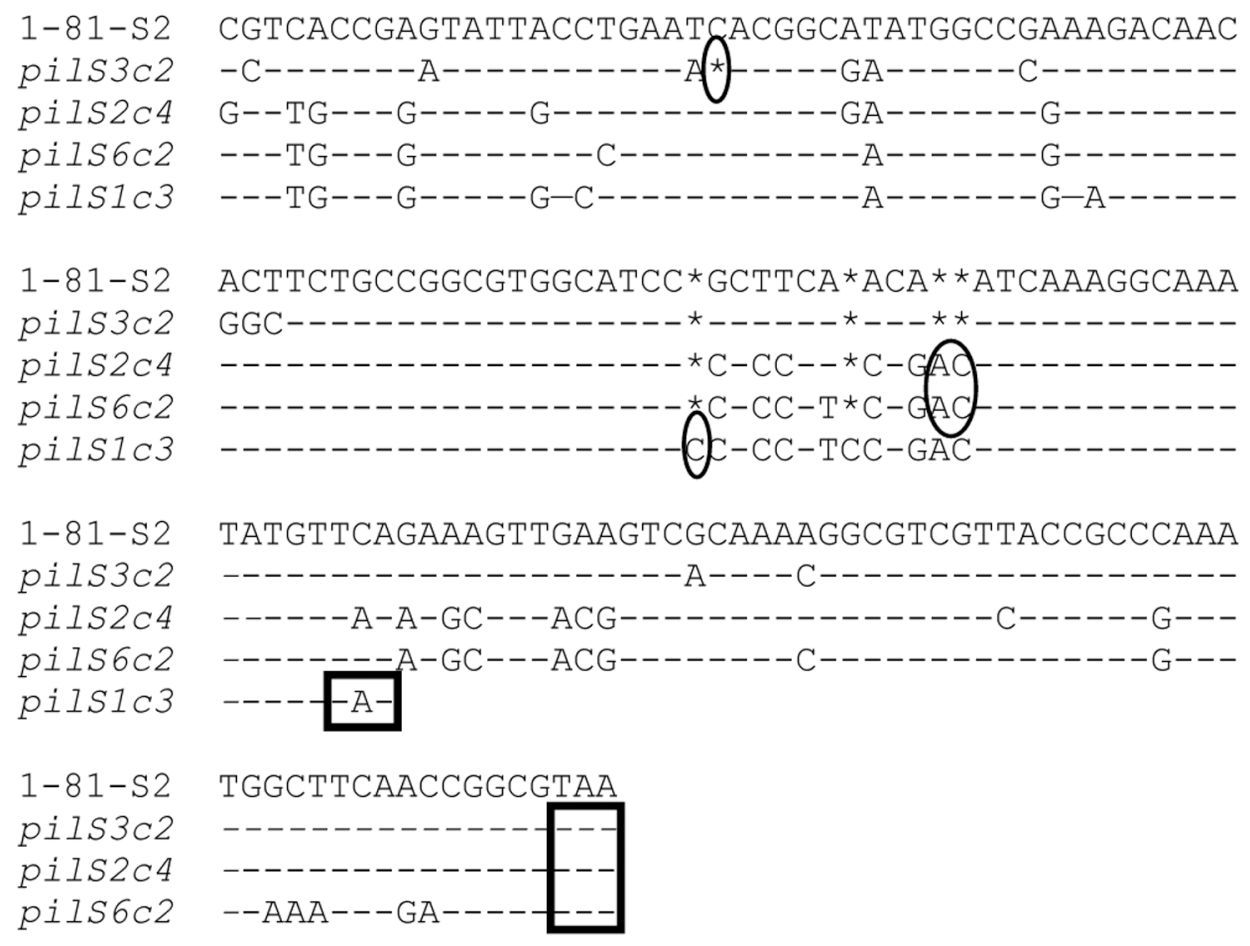

Figure 3. pilS copies in strain FA1090 that produce truncated pilin proteins and confer P- colony morphology

Alignments of pilS copies with 1-81-S2 pilE begin at nucleotide 159 of pilE, numbered from the first nucleotide of the ATG start codon. Sequence divergences from 1-81-S2 pilE are indicated; identical nucleotide to 1-81-S2, (-); nucleotide missing relative to other pilS copies or pile, (*). Frameshifts relative to 1-81-S2 are circled. Each pilS sequence terminates with the predicted stop codon (boxed). 
Table 1

Distribution of pilS copies in variants of FA1090 1-81-S2 Gc

$\mathrm{P}+$ and $\mathrm{P}-$ variants were isolated after passage of FA1090 1-81-S2 and its recA6 derivative, yielding $92 \mathrm{P}+$ and $61 \mathrm{P}-$ recombination events where the donor silent copy could be assigned to one or two pilS sequences. The percent appearance of each pils copy, or sequence corresponding to two pils copies, was determined. Each pilS copy is annotated for its copy number and position in pilS as follows: pilS3cl = pilS locus 3, copy 1 .

\begin{tabular}{|c|c|c|}
\hline \multirow[b]{2}{*}{ pilS copy } & \multicolumn{2}{|c|}{$\%$ appearance in: } \\
\hline & $\mathbf{P}+$ & $\mathbf{P}-$ \\
\hline pilS2c1 OR pilS6c1 & 34.8 & 6.6 \\
\hline pilS3c1 & 21.7 & 1.6 \\
\hline pilS3c2 & 17.4 & 36.1 \\
\hline pilS1c1 & 8.3 & -- \\
\hline pilS2c4 OR pilS6cl & 4.3 & -- \\
\hline pilS3c3 & 3.3 & -- \\
\hline pils1c5 & 3.3 & 1.6 \\
\hline pils2c4 & 1.1 & 36.1 \\
\hline pils1c4 & 1.1 & 4.9 \\
\hline pilS7c1 & 1.1 & 1.6 \\
\hline pilS6c3 & 1.1 & -- \\
\hline pilS2c5 & 1.1 & -- \\
\hline pils6cl & 1.1 & 3.3 \\
\hline pilS2c1 & -- & 4.9 \\
\hline pils6c2 & -- & 1.6 \\
\hline pils1c3 & -- & 1.6 \\
\hline
\end{tabular}

